\title{
Designing the $5 \mathrm{G}$ network infrastructure: a flexible and reconfigurable architecture based on context and content information
}

\author{
Dario Sabella ${ }^{1 *} \mathbb{D}$, Pablo Serrano ${ }^{2}$, Giovanni Stea ${ }^{3}$, Antonio Virdis ${ }^{3}$, llenia Tinnirello ${ }^{4}$, Fabrizio Giuliano ${ }^{4}$, \\ Domenico Garlisi ${ }^{4}$, Panagiotis Vlacheas ${ }^{5}$, Panagiotis Demestichas ${ }^{7}$, Vasilis Foteinos ${ }^{5}$, Nikolaos Bartzoudis ${ }^{6}$ \\ and Miquel Payaró ${ }^{2}$
}

\begin{abstract}
$5 G$ networks will have to offer extremely high volumes of content, compared to those of today's. Moreover, they will have to support heterogeneous traffics, including machine-to-machine, generated by a massive volume of Internet-of-Things devices. Traffic demands will be variable in time and space. In this work, we argue that all this can be achieved in a cost-effective way if the network is flexible and reconfigurable. We present the Flex5Gware network architecture, designed to meet the above requirements. Moreover, we discuss the links between flexibility and reconfigurability, on the one side, and context awareness and content awareness, on the other; we show how two of the building blocks of the Flex5Gware architecture, namely on-the-fly MAC reconfiguration and dynamic multi-cell coordinated resource scheduling, can leverage context and content information to make reconfiguration decisions. Our evaluation, conducted by both prototyping and simulation, show that this brings significant benefits in terms of improved coexistence of heterogeneous access networks and reduced latency. We terminate our paper by discussing how the Flex5Gware architecture aims at influencing future system standardization and leveraging the benefits of some key $5 \mathrm{G}$ networking enablers described in the paper.
\end{abstract}

Keywords: Flexible HW/SW platforms, 5 G networking, Context-awareness, Content-awareness, Reconfigurability

\section{Review}

\subsection{Introduction}

Current mobile networks, from $2 \mathrm{G}$ to recent LTE-advanced systems, have been designed and deployed with a connection-centric mindset. Moreover, they were traditionally conceived and over-provisioned in terms of capacity, in order to handle peak-hour traffic. Mobile operators have been constrained by the inflexibility of their radio access network (RAN) equipment. The latter represents a limitation for both the communication and computation capabilities, due to the lack of reconfigurability, with a negative impact on the cost.

The last few years have witnessed an explosion of the traffic demand (dominated by video content [1]) and a paradigm shift in terms of service usage. Content sharing and social behavior redefined the way the network is used.

\footnotetext{
* Correspondence: dario.sabella@intel.com

'Intel, Neubiberg, Germany

Full list of author information is available at the end of the article
}

The next years will also witness a further, more dramatic increase of machine-to-machine connections [2], due to the progressive introduction of the Internet-of-Things (IoT) traffic and services. Moreover, many delay-critical services will need to be supported by $5 \mathrm{G}$ networks.

Such services will transform several vertical business segments [3] (e.g., automotive and mobility, factories of the future, healthcare, media and entertainment, energy) and will also create new revenue streams for many stakeholders, e.g., operators, vendors, content, and application providers.

A key characteristic of $5 \mathrm{G}$ services associated with content-delivery and IoT, is the variance of their demands, in time and space. Therefore, flexibility and reconfigurability can enable the mobile network to adapt to these variations and achieve cost efficiency, dispensing with the need of planning for worst-case scenarios. However, today's connection-centric mobile networks do not possess the required flexibility to adapt to varying 
traffic needs and to provide content and communication services where and when actually needed. Flexibility and reconfigurability can be achieved through technological improvements enabled by new hardware (HW) and software (SW) platforms. These features should stem from the ability to manage context information, acquired through measurements performed by network equipment and sensors, in order to gain insight over traffic, mobility, users, conditions, and crowds. Thus, the paradigm shift should be towards content- and context-aware networks.

This paper describes architectural features, developed within the framework of the Flex5Gware EU project [4], that incorporate context and content-awareness into mobile networks, with the explicit aim to influence future $5 \mathrm{G}$ systems. We argue that this evolution should capitalize on the availability of reconfigurable radio platforms for the RAN and take advantage of virtualization technologies, such as Cloud-RAN and the standardized network function virtualization (NFV) framework [5], including virtual RAN (vRAN). On top of that, it should adopt software-defined network (SDN) technologies and the recent multi-access edge computing (MEC) [6]. The above are commonly considered as key ingredients of future 5G systems [3] that will provide operators with the required flexibility and reconfigurability to satisfy this increasing traffic demand.

According to the above, the two features described in this paper are as follows:

1. Context-aware reconfigurable radio platform management, through which a flexible, efficient and reconfigurable HW/SW can adapt access network characteristics based on data and context information;
2. Content-aware coordinated resource allocation strategies (implemented in C-RAN/vRAN environments), whereby intelligent baseband processing within vRAN exploits the presence of information related to the aggregation point of several base stations.

These enablers can be realized by exploiting real-time radio network information, gathered through a MEC framework and APIs (possibly co-located with the same virtualized infrastructure hosting vRAN and other virtualized technologies), where context information from the RAN can be provided to user level applications or other services for network performance and quality of experience (QoE) improvements. These enablers, together with the reference system considered in this paper, are depicted in Fig. 1.

We evaluate the impact of our enablers through both prototyping and simulation. An experimental evaluation of a context-aware reconfigurable radio platform shows that even low-level medium-access operations can be easily adapted to specific interference conditions in order to optimize the network capacity. Moreover, we show via simulation that our content-aware coordinated resource allocation allows operators to manage inter-cell interference so that real-time traffic experiences smaller delays, even in congested scenarios, without sacrificing the cell throughput.

The remainder of this paper is organized as follows: the related work is discussed in Section 2; Section 3 provides an overview of the Flex5Gware functional architecture and describes our technology enablers; Section 4 presents and discusses performance evaluation results.

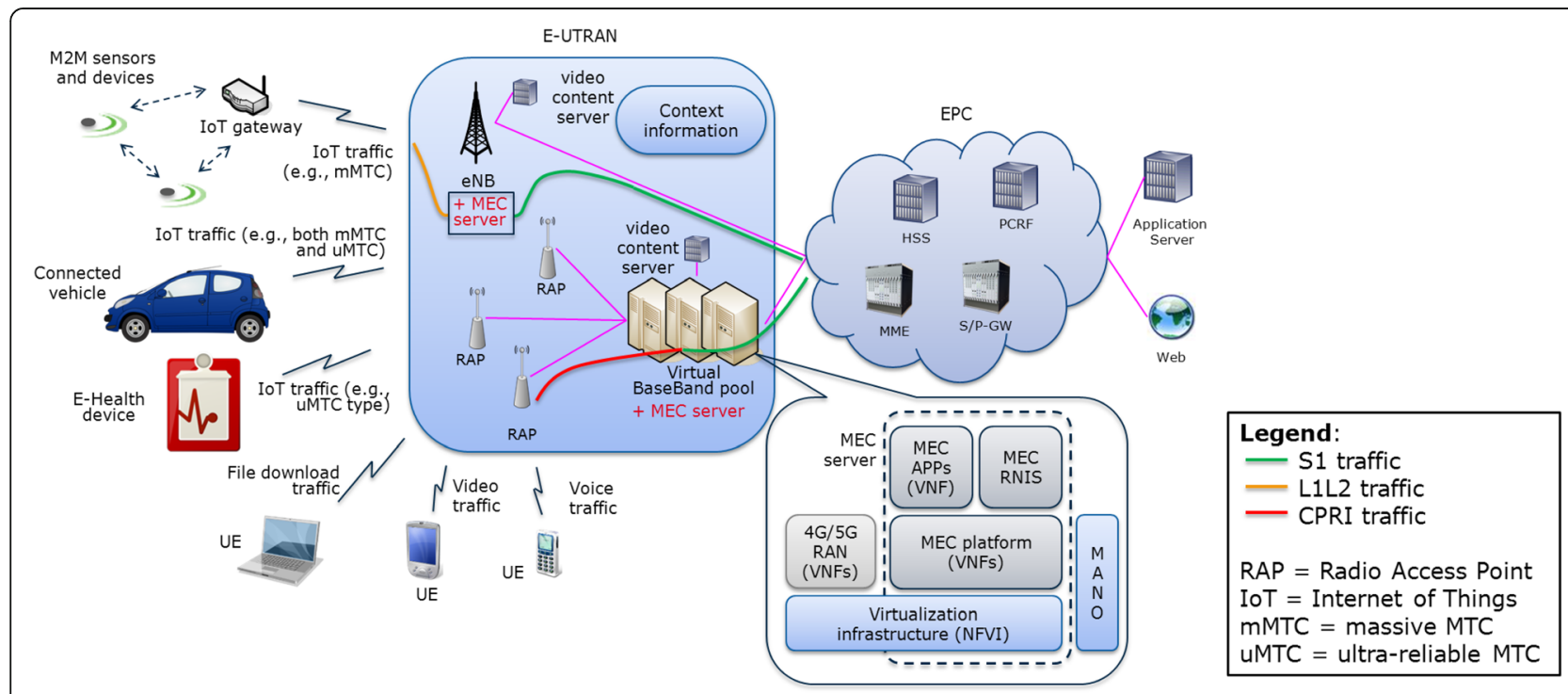

Fig. 1 Exemplary representation of the considered $5 G$ reference system 
Finally, Section 5 discusses implementation aspects and standardization impacts and concludes the paper.

\subsection{Related work}

As it has been pointed out in the previous section, in this paper, we present a novel reference functional architecture, with one of its main features being the integration of technologies associated with the core network (e.g., SDN/ NFV) with others associated with the access network (e.g., flexibility similar to that of software defined radio (SDR)). In the following, we discuss those relevant trends and novel ideas in the design of a 5G network architecture that are most related to our contribution, identifying the similarities and their differences (for a full description of these trends and proposals, see [7]):

\subsubsection{Novel physical network architectures}

Networks will leverage different heterogeneous radio access technologies (RATs), and heterogeneous deployments, in terms of different cell sizes and frequency bands. Furthermore, the distances between the RATs providing access to users and the core network will be heterogeneous as well, from a few meters to several hundred kilometers apart [8]. Given the ability to place networking functionality anywhere in the network (thanks to its softwarization and modularity, as discussed next), depending on the service to be provided and the required KPIs to fulfill, the network could rearrange the functionality in different ways. Our presented functional architecture takes into account this heterogeneity of technologies and deployments and supports contextaware optimization of topologies and resource assignments; however, its focus is on identifying the required functionality to operate such types of networks and the potential benefits, and not the specific interfaces and primitives to support this operation.

\subsubsection{Modularization of the network}

Another trend is the modularization of the architecture. In fact, a general reference scheme was agreed in 3GPP [9], which identifies the building blocks (logical elements) to compose an architecture. The actual definition of these modules and the related interfaces is a difficult ongoing challenge, which needs to address both the degrees of freedom available given the few constraints, and the need to identify the relevant metrics to support a scientific performance assessment of the different architecture. While 3GPP has already proposed a modularized architecture [10] for 5G networking, so far all the considered elements belong to the core network. In this paper, we identify certain SW modules related to the access network, whose modularization (which would bring the corresponding benefits, supporting, e.g., flexible placement) is yet to be tackled.

\subsubsection{Network softwarization}

The design, deployment, and operation of diverse network components become highly flexible when SW programmability properties are adopted. SW facilitates control, management, and on-the-fly reconfiguration of HW infrastructure and devices, guaranteeing effective service development and deployment, as well as rapid adaptation to very demanding and changing contexts of operation, ensuring Quality of Service (QoS) and QoE. In many cases, it allows for sophisticated orchestration tasks by removing complexities and exploiting advanced computation capabilities. A valuable study regarding major principles, enablers and solutions on network softwarization can be found in [11]. In addition, an analysis of existing softwarized mobile network architecture proposals can be found in [12], along with an analysis of benefits but also some open issues.

Our work attempts to exploit the described benefits and to address the raised limitations through innovative SW modules, while also studying interface abstractions and uniform (HW-agnostic) APIs. Based on a SW-based operation (with behavior determined by SW), we achieve improving the programmability and reconfigurability potential of the network elements. On top of that, context information supports taking intelligent decisions such as the dynamic reloading of new SW-coded functionalities. Moreover, softwarization is beneficial for flexibility in terms of activated protocols, as the most appropriate protocol fitting the possibly very specific context at hands could be automatically downloaded upon need.

\subsubsection{Access-agnosticism}

By reducing the dependencies between the core network and the access networks, each one can evolve in a different way, and convergence among 3GPP, non-3GPP, and fixed access networks is enabled. The key motivation behind the design of a core network agnostic to the access technologies is to achieve a future-proof solution. There are basically two approaches to provide this agnosticism in the access: (a) the use of a convergence layer between the two networks or (b) the use of standard interfaces across technologies. While we do not contribute to any of these two approaches, as we do not provide any specific technical solution, our functional architecture enables the operation of access agnostic networks, while our results further illustrate the benefit of partially-reconfigurable radio stacks.

\subsubsection{Orchestration/coordination}

Frequently changing environments are foreseen for the fifth generation of mobile wireless systems. Consequently, nodes/devices are expected to experience an extreme context-dependent behavior. A change in context will result in possible reconfiguration(s) that may 
risk overall performance. Therefore, global acceptable solutions should be pursued to support these reconfigurations and maximize the desired benefits. However, the $5 \mathrm{G}$ ecosystem consists of diverse and heterogeneous devices in terms of purpose, scope, technologies, and vendors/manufacturers. Unavoidably, critical situations will occur, e.g., instabilities, conflicts, and competitions. In this direction, the article in [13] discusses the need for a flexible mobile network architecture that integrates efficiently the many different future technologies into one mobile network. To this end, particular attention is being paid to orchestration and management as an essential component of the mobile network. The proposed approach follows the paradigm of SDN, adopting the SW-defined mobile network control concept (focusing on wireless-specific functions). This solution is specifically devised to control mobile network functionality (both control and data plane functions), which can be placed arbitrarily in the edge cloud or the central cloud. Another approach for a new 5G architecture, called "virtualized cloud radio access network", is presented in [14]. This effort attempts to move towards a cell-less 5G network architecture. In this article, the authors show how coordination and joint optimization of heterogeneous resources can be achieved in 5G transport networks. For this purpose, a joint framework, a virtualized base station that can be formed for either a cell or a user, is proposed for resource sharing and base station coordination.

Our work shares the same goal with the aforementioned studies in terms of orchestrating/coordinating heterogeneous resources. It also follows the SDN paradigm with a multi node coordination layer (acting as a controller) that enables a multi-node technology-agnostic SW operation. As will be presented in more detail in the next section, a core component of this layer is a HW-agnostic SW module that is dedicated to the coordination of the different elements as well as the underlying heterogeneous HW infrastructure's reconfiguration. Its main innovation lies in its capability to exploit high-level policy information (application/user requirements, QoS, energy efficiency, scalability) and lower level context information (traffic levels/ variations, HW capabilities).

\subsubsection{Interworking with non-3GPP networks}

Interworking of cellular networks with other types of access networks has received significant attention from the 3GPP over the past years. There are some notable proposals, such as, e.g., interworking of Hotspot 2.0 with ANDSF, but most of the solutions are basically patches over existing interfaces-this situation, and a simpler management solution, could be enabled with the arrival of SDN and NFV [15]. R15, arrived by mid 2017 [16], already supports a number of integrations between
WLAN and LTE, and the development of Licensed Assisted Access might boost the heterogeneity of deployments, given the diversity in preferences across operators [17]. In this paper, we illustrate the advantages of a flexible operation under some coexistence scenarios, along with the need to design mechanisms for a graceful coexistence of licensed and unlicensed technologies.

Design considerations for a 5G network architecture are also discussed in [18], where intelligent use of network data to facilitate optimal use of network resources for QoE provisioning and planning is particularly analyzed. The work presented in this paper is well aligned with the authors' findings. Also, it is worth mentioning that the 5G Architecture Working Group [19], within the 5GPPP Initiative, is considering capturing novel trends and key technological enablers for the realization of the $5 \mathrm{G}$ architecture. Our findings were valuable contributions to the efforts of the above working group.

Finally, this paper extends and completes our preliminary work [20]. Besides a revised presentation, which frames our contribution into the context of the related work on the topic, this paper includes a more detailed performance evaluation, with novel results.

\subsection{Methods}

In this section, we first introduce the functional architecture that serves as a reference for our contributions, which was developed within the Flex5Gware 5G PPP project [4]. We then describe in detail two building blocks of the Flex5GWare architecture, namely the context-aware reconfigurable radio platform and the content-aware coordinated resource allocation strategies.

\subsubsection{Reference functional architecture}

The functional architecture is illustrated in Fig. 2 and relies upon three key concepts: flexibility, reconfigurability, and monitoring. Our approach combines key contributions from other $5 \mathrm{G}$ initiatives, aiming at the integration of SDN/NFV [13] or RAN [21] in a common architecture. Flex5Gware focuses on integrating the above three key concepts, traditionally associated with cognitive radio [22], into a $5 \mathrm{G}$ reference architecture. The functional modules are classified into three layers, depending on their focus, namely, (i) the design of a single device (node operation), (ii) the coordination of devices, and (iii) the control and optimization of the network (intelligent programs).

Considering node operation (i), the most relevant concept is flexibility: in order to cope with the high variability of $5 \mathrm{G}$ application requirements and network topologies, 5G technologies will support advanced reconfiguration capabilities at both the PHY and mediumaccess levels. To take advantage of this flexibility, a sensing functionality is required, which eventually is responsible 


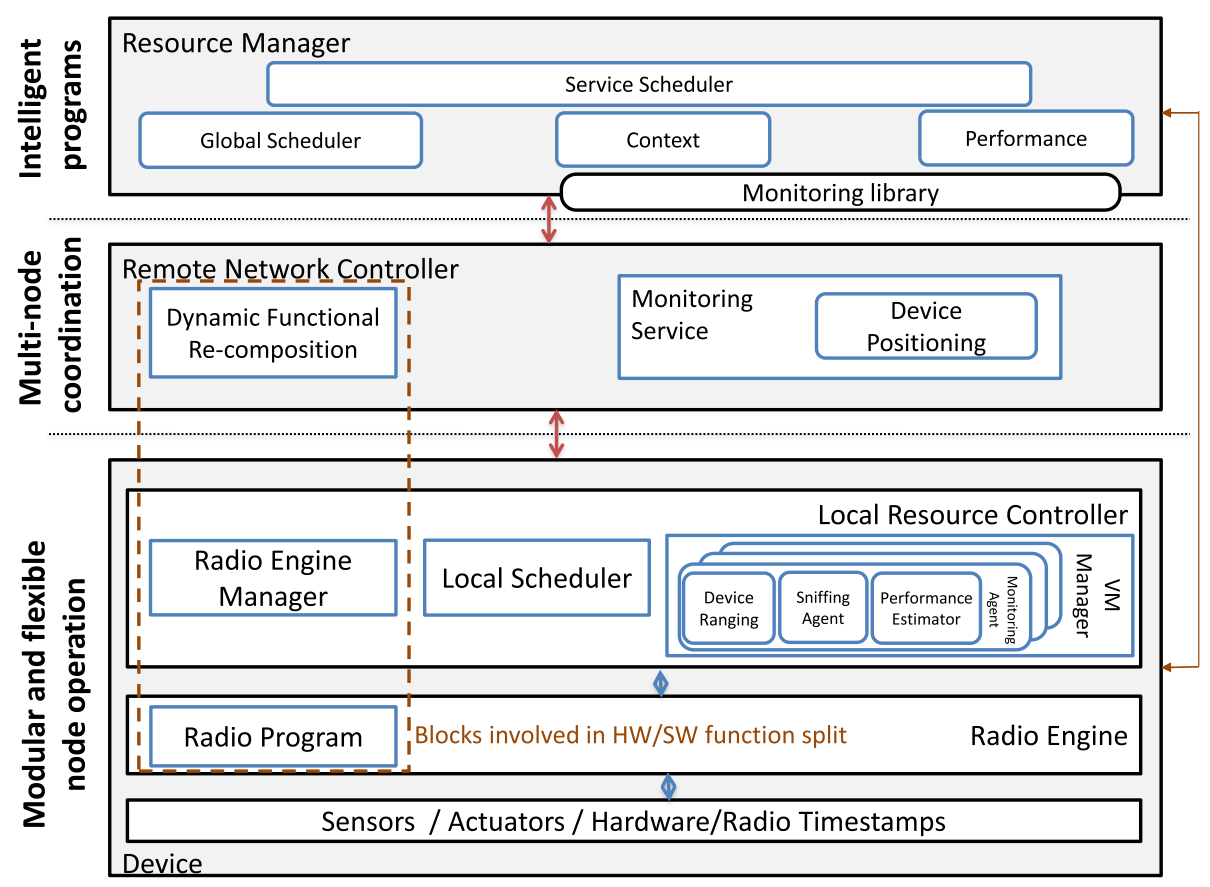

Fig. 2 Functional architecture for context and content-aware 5G platforms

for gathering the information that will trigger the reconfigurations of the node operation. This sensing functionality is designed building on "monitoring agents," which abstracts heterogeneous sensors and exposes their measurements, and can run as virtualized programs that are provisioned on demand (thanks to virtualization techniques, the same set of $\mathrm{HW}$ primitives can be shared by multiple agents). Key sensing functionality, as illustrated in Fig. 2, includes performance estimators, sniffing agents, and device ranging to support accurate localization, which enables context-aware optimizations even for indoor scenarios.

Our architecture moves from the traditional approach of "one-size-fits-all" protocol MAC/PHY stack to an innovative paradigm of on-the-fly configuration of context-specific stacks. This is achieved thanks to a flexible MAC architecture, which describes the interfaces allowing for MAC reconfiguration of terminals, followed by dynamic, flexible, and fast reconfiguration of the PHY (e.g., the RF bandwidth). Together, these two capabilities provide a fully flexible and reconfigurable radio interface so that upper layers can sit on top, independent of both PHY and MAC layers. These reconfigurations are enabled by the design of wireless devices based on a radio engine, which is a generic executor of radio programs that may implement completely different protocols. The orchestration of these programs is performed by a radio engine manager, responsible for the scheduling and installation of the radio programs in the engine. Finally, a local scheduler is responsible for managing the resources provided to a user by manipulating the allocation scheme.

The intelligent programs layer (iii) consists of modules that optimize network performance, based on an information-centric operation and exploiting the reconfigurability and monitoring features. The monitoring library is an API that provides access to the data collected by sensors and monitoring agents throughout the network. In the envisioned 5G ecosystem, monitoring of network elements and devices will be of paramount importance. However, a single mechanism would be insufficient to cover all scenarios and requirements. Therefore, the SW framework is flexible enough to allow the incorporation of different solutions that target different goals. This monitoring library feeds two modules that extract network-wide performance and context estimations, which in turn trigger different types of optimizations. A global scheduler (GS) scheme allows nodes to share information about users' resource allocation requests, so that resource allocation schemes can be decided globally and applied. A performance-aware optimization module aims at enhanced performance while reducing the energy consumption, taking as input highlevel policy information and lower level context information (e.g., traffic levels/variations, HW capabilities, capacity), and its outcome results in the direct reconfiguration of individual resources or the management and configuration of multiple resources, using a multi-objective optimization algorithm.

For instance, performance degradations or context variations may trigger the activation of additional network 
elements or switching to a RAT that is more robust. Moreover, the GS coordinates the operation of the base stations, enabling the C-RAN and vRAN vision as described next, and a service scheduler that coordinates the optimization of all these elements, to enable a smooth operation precluding conflicts.

The node operation (i) and intelligent program (ii) layers are connected by the multi-node coordination (ii), whose goal is to design and implement the required control and management plane to enable a multi-node technology-agnostic SW operation, i.e., develop the tools required to coordinate in real-time the different SW modules available across multiple nodes. It is composed of: (a) a monitoring service, which gathers data from the local monitoring agents of the multiple devices (including sensors), processes their information and forwards it to the intelligent programs via the monitoring library described above and (b) a dynamic functional re-composition, which stitches together SW and HW functions and abstracts the changing of the operation of multiple devices to the performance optimizers, hence providing a technology-agnostic reconfigurability service to the intelligent programs. Its core operation is based on a context-aware, cognitive, and dynamic HW/SW partitioning mechanism for $5 \mathrm{G}$ network elements and devices. This mechanism exploits knowledge (e.g., prediction of a hotspot) derived by network and sensor measurements and decides upon the HW or SW execution of functions (e.g., LTE, 3GPP-based PHY/ MAC functions) in order to fulfill and maintain the application goals. It receives requests for reconfiguration from the intelligent programs accompanied with useful information (e.g., policies, context), triggers the HW/SW partitioning mechanism to identify possible functional compositions compliant to the request, decides the optimal one, and sends the decision to the local resource controller for the execution of the appropriate actions.

As an example of field-use of the proposed architecture, content-based system requirements could trigger a network-wide partitioning and reconfiguration of HW-accelerated (HWA) and SW baseband functions, tailored for different traffic service delivery and QoE needs. In Fig. 3, it is shown that out of the different available options, such flexible function splits (FSs) could result in placing (1) the hotspot's (i.e., eNB) layer-2 and above together with the EPC functions in the Cloud (e.g., S1 traffic), (2) the eNB layer-2 and above functions at a local MEC server (L1L2 traffic), or (3) move the entire eNB stack in the Cloud (CPRI traffic) and transform the eNB to a remote radio head (RRH). This dynamic split and reconfiguration of baseband functions is complementary to the partitioning of the eNB protocol stack, either at stack or algorithm level that was promoted relatively recently by key industry actors [23].

We now describe our two key enablers, namely a context-aware reconfigurable radio platform and a content-aware coordinated resource allocation strategy, together with a clear comparison against the stateof-the-art methods. For each introduced enabler, related subsections of Section 4 will describe their performance evaluation results coming from simulations

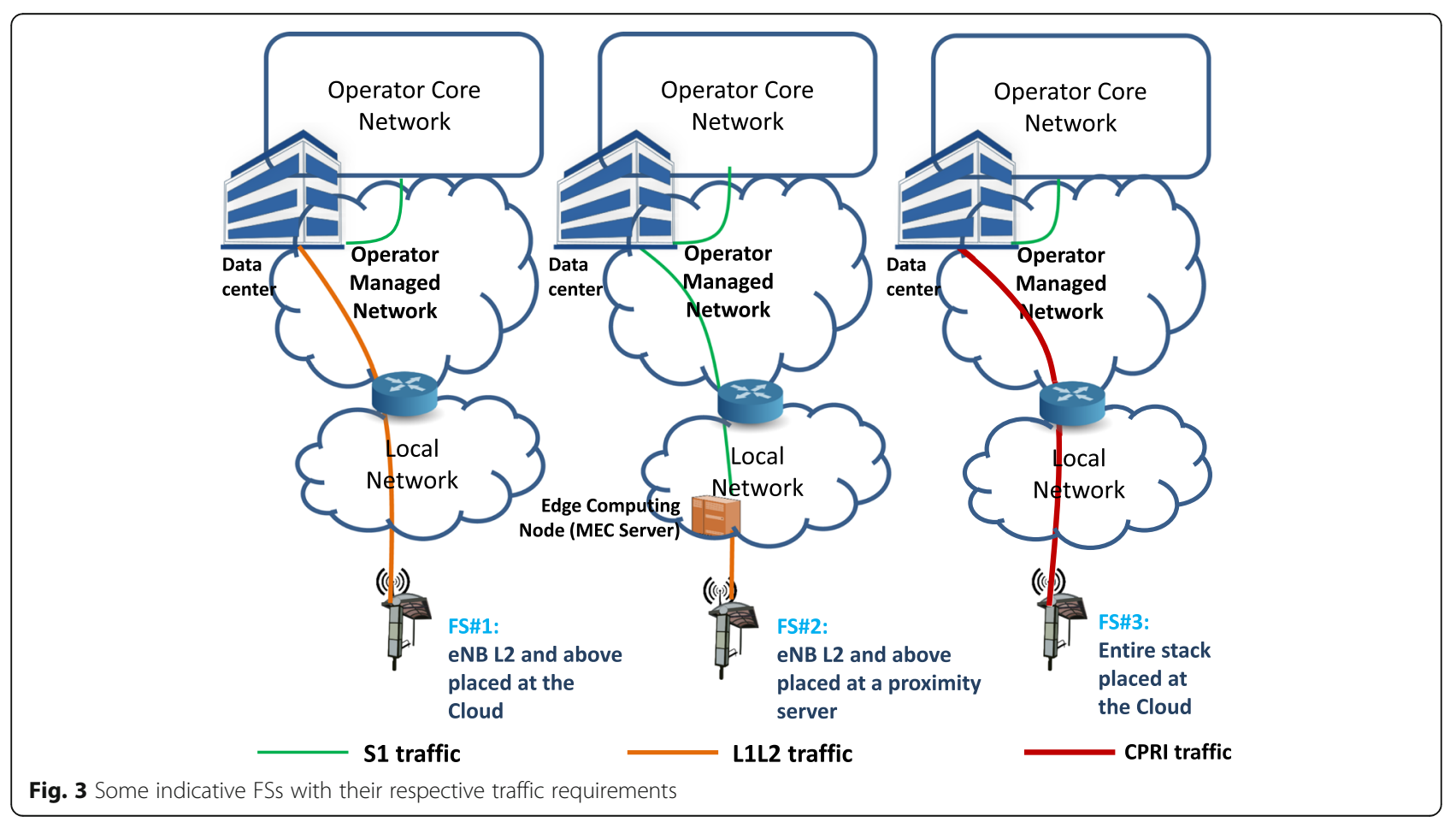


and experimental works, carried out in the framework of the Flex5Gware project.

\subsubsection{Context-aware reconfigurable radio platform}

Device flexibility allows the (re)configuration of contextspecific stacks on the fly, based on context-related triggers. Thanks to the radio engine of the Flex5Gware architecture, reconfigurations can also involve medium access and transceiver operations defined in radio programs, which combine a set of HW primitives and control the behavior of HW devices, e.g., analog front-ends, converters, mixers, filters, base-band modulators, transmission queues, and sensors (like accelerometers or cameras). Radio programs are built similarly to computer programs, sent over the air to the radio engine manager, stored in an internal memory, and changed over time. Changes can be triggered not only by traditional performance-related metrics such as delay or bandwidth, or PHY parameters such as signal strength, but also by context estimators (e.g., weather information, accurate localization including indoor/outdoor information, relative position of the mobile to the user) and other new performance-related metrics, such as energy consumption of HW elements and CPU usage.

The feasibility of this approach has been recently demonstrated by some preliminary work on programmable radio platforms, such as the wireless MAC processor architecture (WMP) for WiFi commercial cards [24] and the TAISC architecture for ZigBee sensor nodes [25]. Flex5Gware extends these solutions not only in terms of more advanced HW primitives but also in terms of monitoring and context classification capabilities.

To demonstrate the capability of adapting low-level medium-access operations in Flex5Gware nodes, we consider a simple reference scenario, in which device-todevice links employing a contention-based protocol, such as the WiFi distribution coordination function (DCF), are activated in areas where sensors are densely deployed and generate periodic traffic. We also assume that sensors transmit to a base station by means of a scheduled-based protocol, such as the LTE one, but channels are allocated in unlicensed bands to offload the cellular network. We also assume that, according to the rate of the sensor data, the frame allocations used for sensors transmissions can be interleaved with empty (blank) frames, as considered in [26].

In the presence of periodic interference sources, DCF legacy devices suffer from severe collisions, because it is very likely that they initiate transmissions that will be disrupted by (non-channel sensing) sensors later on. The higher the frequency of periodic interfering transmissions, the higher the collision probability. DCF devices react to collisions by increasing the contention windows, thus reducing the channel access rate. If DCF devices employ rate adaptation, they will also aim for lower rates, thus increasing the transmission times and, accordingly, the number of collisions.

While DCF devices cannot detect the cause of performance impairments and, in any case, cannot adapt for improving coexistence with periodic interference, flexible 5G nodes can easily predict the interference sources in a given geographical area (e.g. by means of positioning data of the sensors) and modify accordingly the PHY/MAC protocol to be used for device-to-device (D2D) links. For instance, exponential backoff and rate adaptation can be disabled, or customized protocols can be downloaded to the devices to further optimize their performance. Figure 4 shows the functional blocks involved in such an adaptation for a specific radio engine implementation and set of radio primitives based on the WMP architecture [24]. A context monitoring service can be defined by exploiting the data provided by the HW primitives, such as the low-level channel sensing traces, or the ranging measurements towards coexisting devices. Upon detection of predictable interference

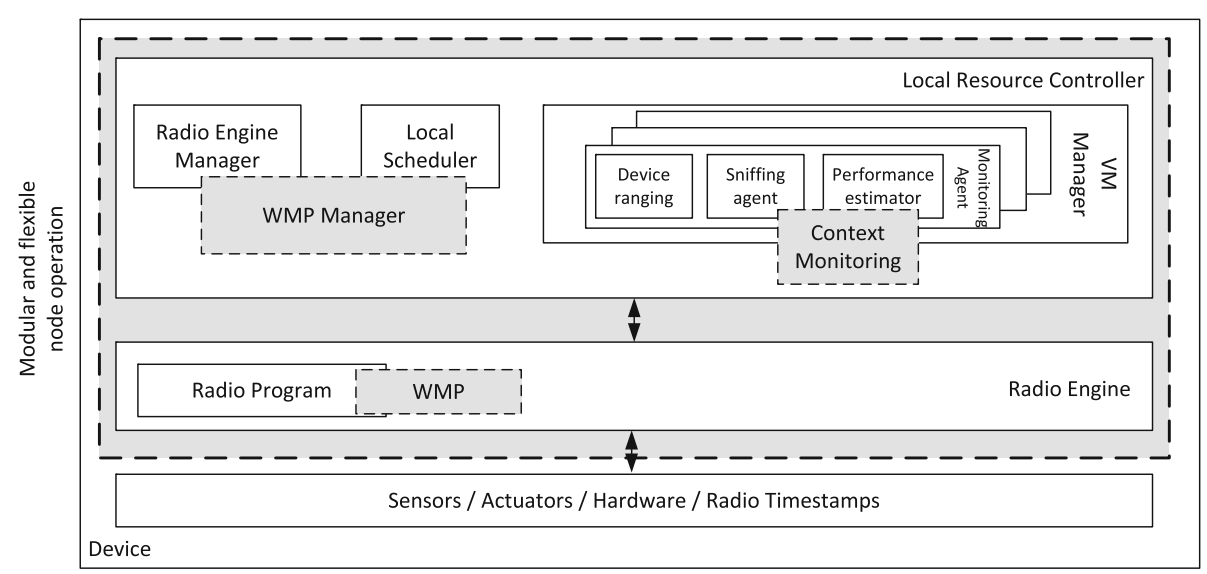

Fig. 4 Placement of the reconfigurable radio platform functions within the reference architecture of Section 3.1 
patterns, the monitoring service can send an input to the radio engine, which in turns can trigger the switch to a new radio program. A radio program is an extended finite state machine composing the HW primitives according to a specific protocol logic.

An exemplary radio program designed for improving coexisting with periodic interference source, called Future NAV (FNAV), modifies DCF by forbidding to start a transmission that cannot be completed and acknowledged before the start of periodic interference interval, which takes place at predictable times. DCF devices will simply consider the medium as virtually busy during these intervals, thus avoiding collisions.

Although the proposed protocol is not the only possible solution, the main novelty of our approach is the possibility to implement cooperation mechanisms between heterogeneous network solutions, based on radio flexibility. This is quite different from current approaches, for example, in the coexistence scenario between LTE in unlicensed bands and WiFi, where the utilization of bandwidth reduction [27], duty-cycling muting, or blank subframes [26] is unilaterally taken by LTE, while the implementation of listen-before talk before transmitting LTE frames requires to build new LTE radio [27, 28].

\subsubsection{Content-aware coordinated resource allocation strategies}

A fundamental research challenge for $5 G$ networks is to devise optimal resource allocation strategies that consider both content and context information. Two paradigms are emerging in this context as possible solutions for the above challenges, namely C-RAN [29] and MEC.

The C-RAN architecture adopts a functional split of the LTE RAN, splitting the eNB into two main elements: a first one, called base-band unit (BBU) implements upper layer functionalities, whereas the RRH deals with radio frequency operations. RRHs are still deployed as radiating elements in the coverage area. BBUs instead can be deployed within a cloud infrastructure at the edge of the network, forming a BBU pool and possibly serving a large area composed of several RRH.

C-RAN is extremely promising in the context of $5 \mathrm{G}$ networks, as it allows dynamic management of computational resources. For example, in C-RAN, computing power can be allocated dynamically to cells on demand and according to their load. Thus, it is suitable to support the $5 \mathrm{G}$ requirement of extreme flexibility. Moreover, in C-RAN environments, issues such as spectral-efficiency optimization and interference reduction, traditionally addressed through HW solutions, can be tackled via a new, entirely SW-based approach, using efficient algorithms which leverage the power of cloud computing.

For instance, dynamic interference coordination at fast timescales is now made challenging by the need to transfer state information among geographically sparse entities (i.e., eNBs and a possibly co-located controller), due to the latencies involved in inter-cell communication [30]. The same information would travel considerably faster as interprocess communication among BBUs in a cloud, making fast-paced algorithms practicable. Moreover, the most recent coordination algorithms require the solution of complex optimization problems, typically belonging to the class of integer linear programming problems. Despite being known to be NP-hard, such problems can be still solved at optimality using state-of-the-art solvers, given enough computational resources [31, 32]. The C-RAN architecture fits naturally in this scenario, providing access to a large and configurable computational capacity, thus enabling the execution of complex algorithms.

On the other hand, C-RAN aggregation points in the network are natural candidates to hosting MEC platforms [33]. The latter can leverage a huge amount of information related to traffic flows, network status, and radio conditions, so that MEC applications can monitor continuously all the contents that are delivered in the network. An exemplary deployment of a MEC platforms and a BBU pool is shown in Fig. 5. More details on the concept of MEC are provided in Section 5.

Furthermore, in order to tackle the increased C-RAN traffic requirements of envisioned content-aware $5 \mathrm{G}$ use cases, flexible RAN FSs have been proposed as an alternative new type of virtualization. By moving and placing cloud-based BBU functions close to, or at the RRHs, the traffic between BBU and RRH is reduced, without affecting the previously mentioned advantages of classic C-RAN. More recently, various FSs at PHY, MAC, RLC, and PDCP layers have been proposed [34]. MEC could also form an integral part of flexible FSs (as seen in Fig. 3). Run-time reconfiguration from one FS to another can be seen as a powerful enabler of content-aware coordinated resource allocation strategies. For instance, switching from a classic C-RAN to a FS where the PHY layer is placed at the $\mathrm{RRH}$, whereas upper hotspot layers

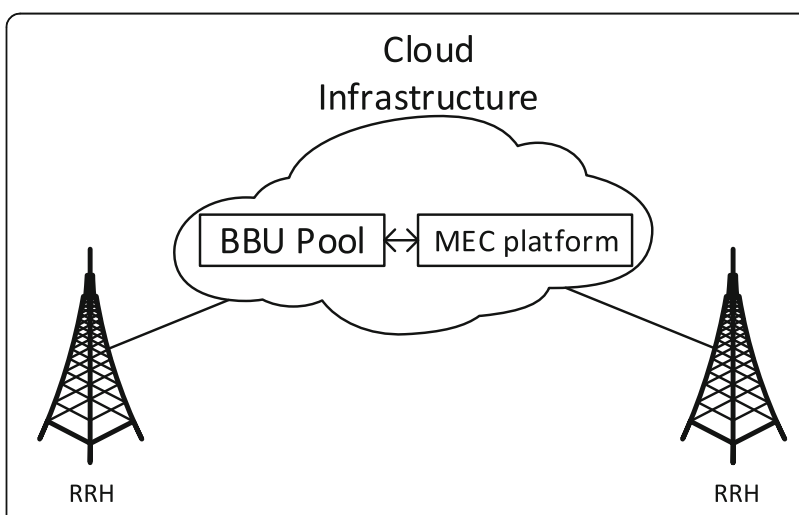

Fig. 5 Exemplary deployment of a MEC platform in a C-RAN architecture 
are placed at a neighboring MEC node could satisfy different key performance indicators (KPIs) related to content-aware demands. Towards this end, a real-time experimental platform was built (Fig. 6) within the Flex5Gware project, featuring all the necessary SW and HWA primitives to allow applying FSs and bandwidth adaptation [35].

In this context, content-aware algorithms, provided with access to such a large and diverse set of information and flexible RAN configuration options, may warrant significant performance benefits, something that could be hardly performed without 5G's architectural advancements, such as C-RAN and MEC. We describe a content-aware algorithm for interference coordination, based on the concept of Coordinated Multi-point (CoMP) algorithms. CoMP has been studied in the literature as an interference-mitigation technique. Among CoMP techniques, Coordinated scheduling (CS) focuses on coordinating the allocation of the physical resource blocks (PRBs) among eNBs [36, 37]. CS can be done statically, regardless of traffic variations [38], or dynamically, by having a coordinator arbitrate resource requests from the coordinated eNB based on their current traffic [31]. A dynamic approach is the one pursued within Flex5Gware, where such coordinator is in fact the global scheduler (GS) mentioned in Section 3.1. Figure 7 shows how the architectural building blocks of Fig. 3 interact towards CoMP CS. More specifically, the local scheduler within the node layer send their scheduling requests (SR) to the monitoring library (ML) on each transmission time interval (TTI). The SRs include the amount of RBs that are requested by the nodes to serve their user equipments (UE) in the downlink. The ML stores the SRs, and the GS fetches a moving average of the SRs periodically (e.g., on a window of $100 \mathrm{~ms}$ ). Based on the latter, the GS computes the amount and placement of RBs for the coordinated nodes and communicates it to them through scheduling commands, which are binary masks stating which RBs a node can/cannot use [31]. The set of nodes coordinated by the same GS is decided at longer timescales (i.e., tens of minutes), by a global power manager, which decides which local nodes should be powered on when, to ensure optimal coverage at minimum energy cost. The part of this framework conceptually belonging to the intelligent program layer is meant to be implemented leveraging MEC and C-RAN technologies.

A coordination policy, i.e., the algorithm run at the GS, affects the performance of specific sets of users differently; hence, the ability to select and change this policy flexibly becomes a key point to improve the user QoE. Context information such as the position of user, or content information such as the one collected by the MEC platform, e.g., the characteristics of data traffic for a particular hotspot (or group thereof), can help improve the performance in this respect. None of the works we are aware of considers context information within their algorithms.

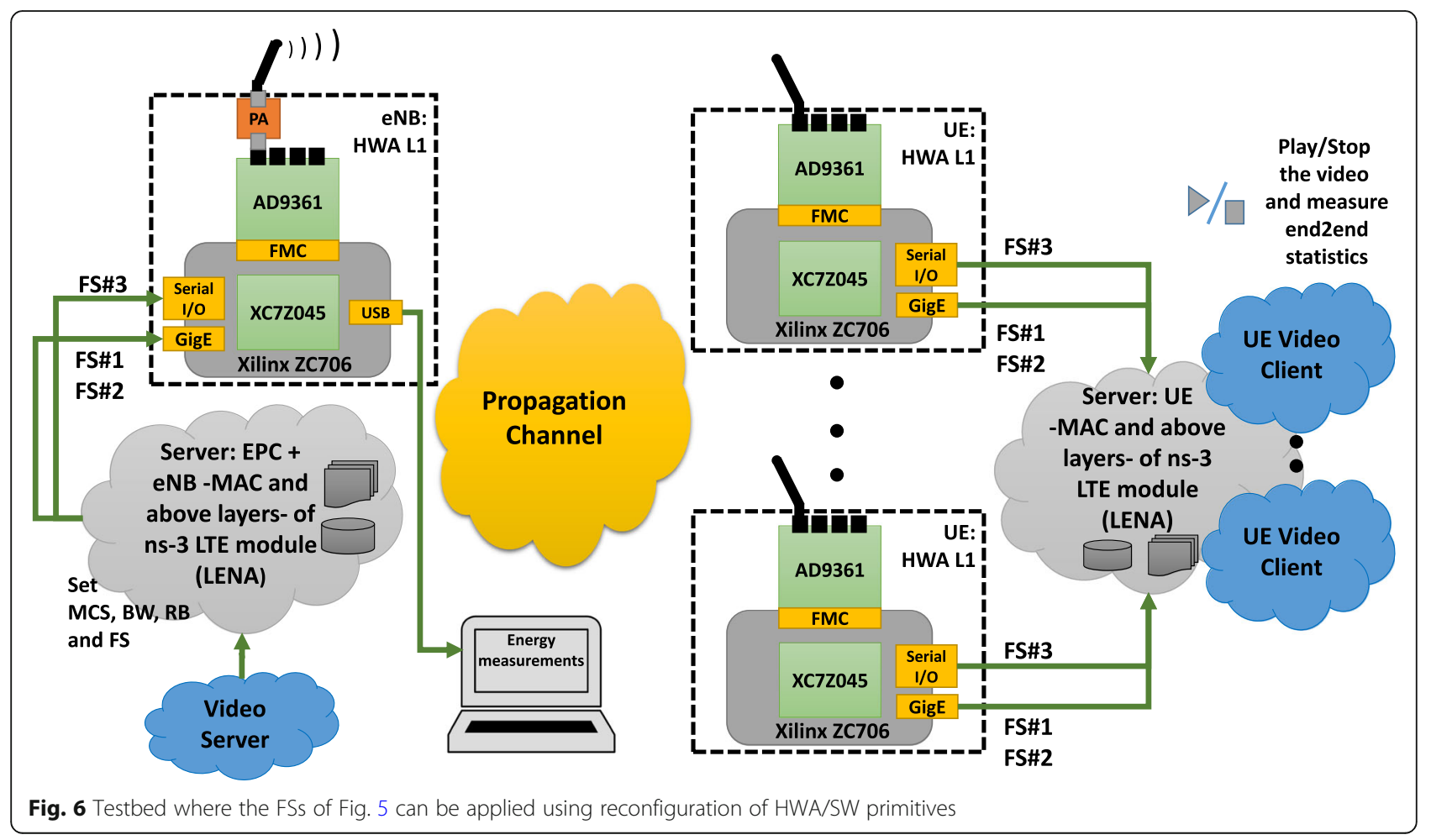




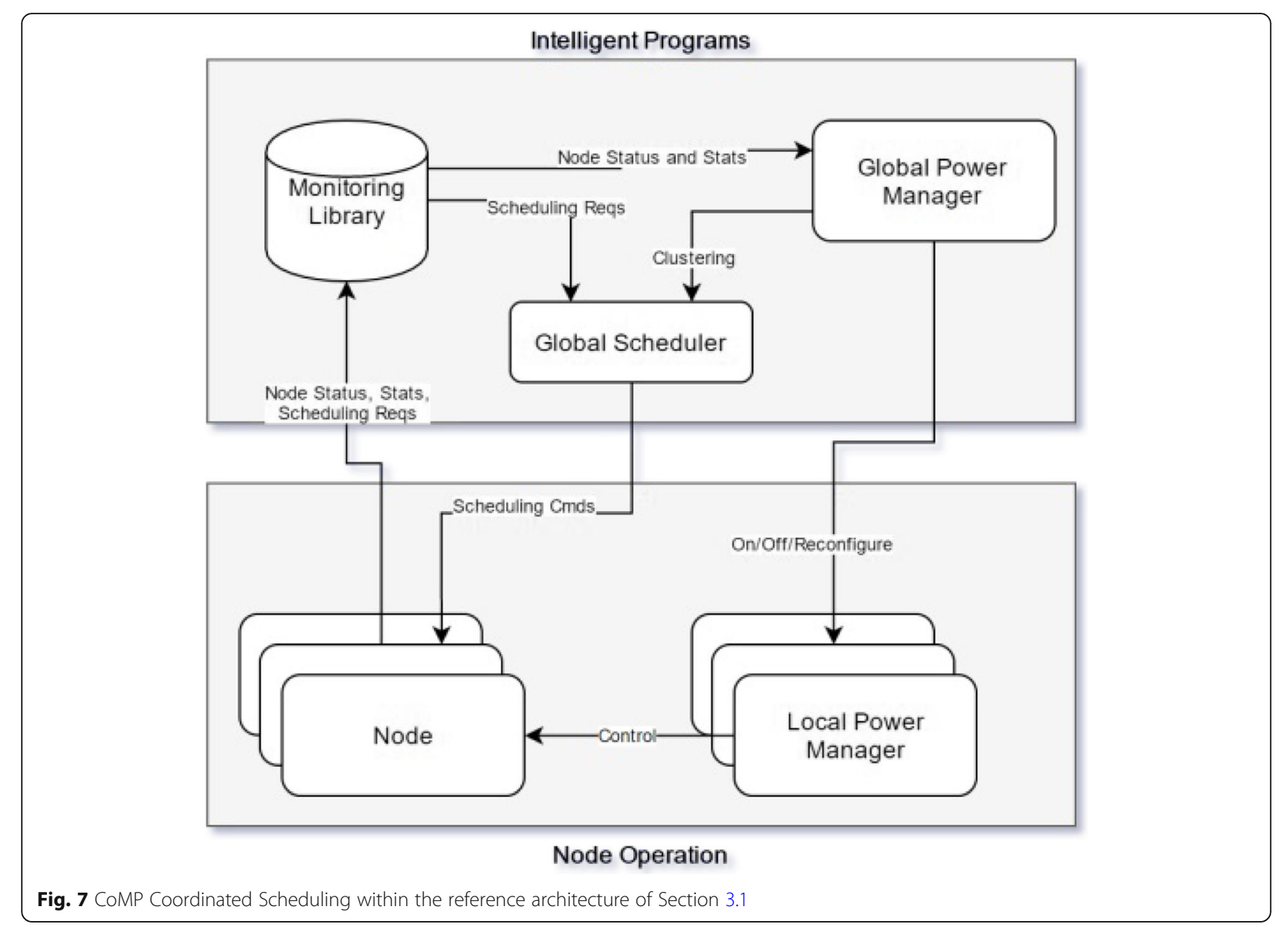

Our content-aware CoMP (CoCo) algorithm exploits knowledge of the traffic content at a hotspot to include QoE considerations in the interference coordination process. When using a classical coordination algorithm, every eNB $x$ belonging to the set $E$ of coordinated eNBs sends its $\mathrm{PRB}$ requests $R_{x}$ to the coordinator, which partitions resources proportionally to the initial requests only. This way, each eNB gets an amount of mutually exclusive RBs computed as:

$$
B_{x}= \begin{cases}R_{x}, \forall x \in E & \sum_{i \in E} R_{i} \leq \text { TotRBs } \\ B_{x}=\frac{R_{x}}{\sum_{i \in E} R_{i}}, \forall x \in E & \text { otherwise }\end{cases}
$$

where TotRBs is the system capacity expressed in PRBs. This occurs at every TTI.

The CoCo algorithm, instead, first collects the requests from each coordinated node, then gathers content information associated to such requests and gives a configurable allocation boost to the ones related to a specific content type. More in detail, assume we have two sets of
eNBs: $H$, composed of those within the hotspot, and $E$, the rest of them. Similarly to the previous case, every eNB $x$ belonging to either set, on each period, sends its PRB requests $\hat{R}_{x}$ to the coordinator. The latter will adjust the requests as follows:

$$
\begin{aligned}
& \hat{R}_{x}=R_{x}, \forall x \in E \\
& \hat{R}_{x}=R_{x} \times \alpha, \forall x \in H
\end{aligned}
$$

where $\alpha$ is the boost provided to the hotspot. The coordinator will then compute the proportional allocation using $\hat{R}_{x}$ in place of $R_{x}$ in (1).

As an example, let us assume a scenario where a crowd is moving towards a stadium, requesting a real-time augmented reality service, i.e., low bandwidth and strict delay requirements. In this case, the stadium hotspot is surrounded by macro cells generating high-bandwidth background traffic, which therefore generates larger resource requests to the coordinator. A content-unaware CoMP would base its decisions on traffic volumes only, thus penalizing the hotspot. A CoCo approach instead can be configured to boost the requests from the hotspot, thus protecting its performance. Using this approach, the interference among 


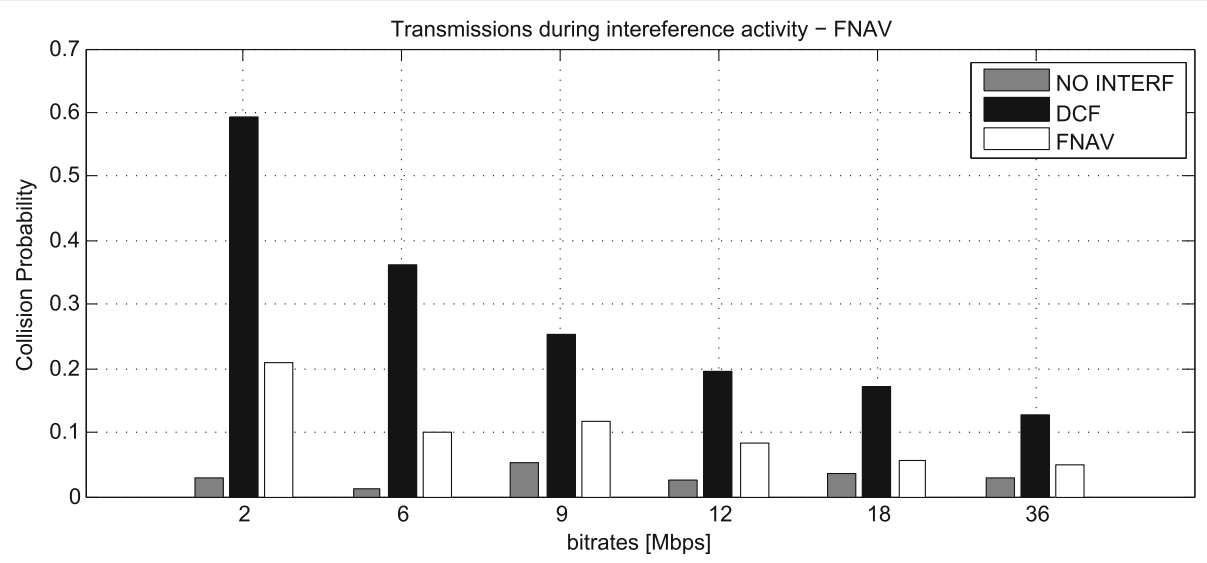

Fig. 8 Collision probability for different values of the bit rate

eNBs will be managed effectively, and a low-delay content access will be guaranteed to users under the hotspot at the same time.

\subsection{Results and discussion}

This section describes some reference scenarios for which Flex5Gware adaptation capabilities have been showcased in real experiments or in simulations.

\subsubsection{Context-aware reconfigurable radio platform}

We consider futuristic scenarios in which wireless nodes do not employ a pre-defined wireless technology rather they rely on a programmable architecture over which completely different protocol stacks can be instantiated, including the stacks of currently standardized technologies. In such a scenario, two devices which need to share some files can be configured, by means of a default cellular technology, for running a WiFi stack in unlicensed bands. The corresponding radio program, if not available, can be uploaded over the air (by using the default cellular technology) before the cellular base station sends the switching command to the new stack.

Assume now that a set of sensor nodes based on the same programmable architecture has been configured with a LTE-like stack to work in unlicensed bands, for transmitting data relevant to some monitoring services. Such a choice permits to offload the cellular network, while supporting long-range links and periodic allocations

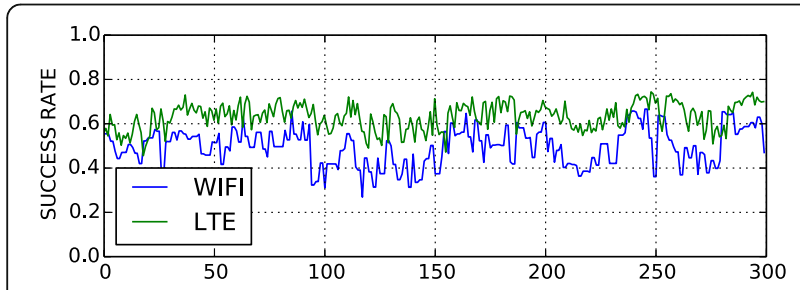

Fig. 9 LTE-U (6 ms activity, 4 ms of silence) + WiFi Legacy DCF of transmission blocks. When the WiFi nodes move in proximity of the sensors, they are able to detect the presence of sensors and their periodic interference pattern and to switch to a context-specific radio program dealing with periodic interference, such as the FNAV radio program presented in Section 3.2.

To demonstrate the feasibility and the effectiveness of configuring context-specific protocols, we run an experiment in which a generic interference source has been configured with a period of $20 \mathrm{~ms}$ and an activity duration of $10 \mathrm{~ms}$ in proximity of a device-to-device link based on our adaptable architecture. In case of legacy access rules, the devices would experience deterministically one or more successful data transmissions (depending on the employed data rate) and a subsequent collision in each interferencefree interval. Instead, as shown in Fig. 8, the FNAV radio program is able to prevent these collisions (thus saving energy and channel time).

Note that the periodic interference source can be due to LTE-U nodes with blank sub-frames, with a solution similar to the one provided in [26]. The number of blank sub-frames can be also adjusted dynamically. Figure 9 shows the success rate of packet transmissions for a WiFi link coexisting with an LTE-U link, when no blank sub-frame is used. It is evident that both technologies suffer for the presence of the other one, despite the differences in terms of transmission bandwidth and channel access scheme. Indeed, for LTE links, the presence of

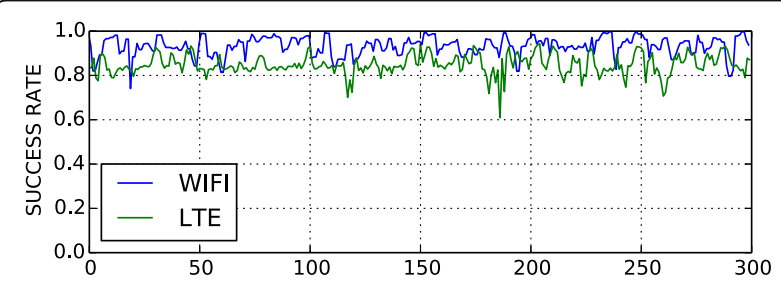

Fig. 10 LTE-U (6 ms activity, 4 ms of silence) + WiFI WMP TDMA 


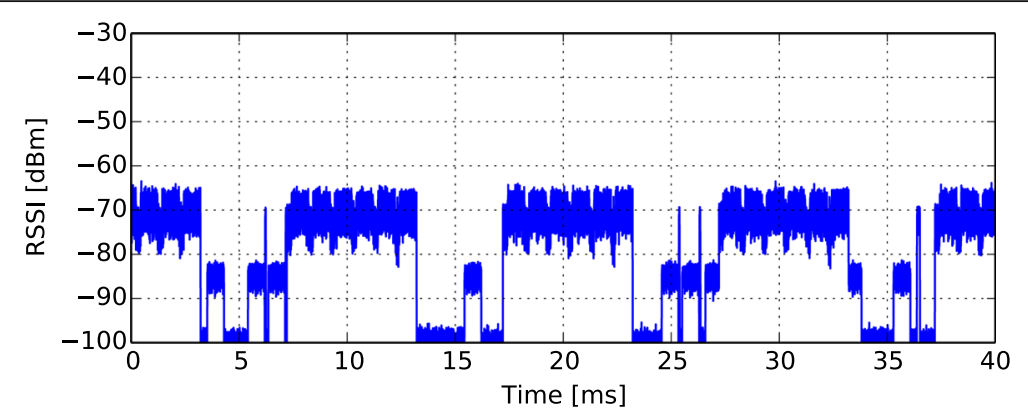

Fig. 11 WiFi DCF transmission over LTE-U pattern

a random-access interferer can destroy synchronization frames, which results in the loss of several transmission blocks. On the other side, for WiFi links, the simple CCA mechanism is not sufficient to avoid channel interference because, after a transient phase, the device adjusts the estimation of the noise level by assuming that LTE frames are due to background noise and therefore by considering the channel as idle during LTE transmissions.

If blank sub-frames are employed, even when such a noise level adjustment does not occur, the periodic scheduling of LTE active sub-frames collides with ongoing WiFi transmissions, which will likely experience other collisions during the LTE frames and increase the backoff counter accordingly, thus resulting in a reduction of the channel access rate.

If a FNAV mechanism is employed, periodic collisions can be avoided, thus allowing WiFi stations to access the channel effectively (i.e., without large contention windows) during the blank sub-frame. A critical issue is keeping synchronization between the expected start of the LTE active sub-frames estimated by WiFi nodes and the real-LTE transmissions. WiFi nodes can adjust this expected start by means of the real carrier sense function, whose results can be compared with the virtual carrier sense results set by FNAV.

Figure 10 shows the success rate experienced by WiFi nodes when FNAV is used and LTE active sub-frames last 6 ms: the results clearly show a significant improvement in terms of packet success delivery.

Finally, Figs. 9 and 10 below visualize the phenomena characterizing the coexistence between $\mathrm{WiFi}$ and LTE links in case of legacy DCF and FNAV, by plotting the RSSI values measured by a monitoring SDR node during a channel access phase of 40 ms: different RSSI values correspond to different transmitters. From the occupancy patterns (idle/busy times) we can easily recognize LTE transmitters (i.e., contiguous intervals of activity lasting $6 \mathrm{~ms}$ ) and WiFi data and ack transmitters (small activity intervals with random idle times, followed by a spike corresponding to the short time required for ack transmission).

In Fig. 11 (legacy protocol), it is evident that the LTE blank sub-frames can be wasted because of the random backoff with increased contention windows, while in the second scenario (Fig. 12) WiFi transmissions are efficiently packed within the available time interval.

Both proposed scenarios describe how enhanced information offered by network cards can avoid significant interference problems and cross-technology coexistence.

\subsubsection{Content-aware coordinated resource allocation}

We now show how content information, obtained exploiting the future $5 \mathrm{G}$ architectural enhancements, e.g., C-RAN and MEC, are used by our CoCo approach. We demonstrate via simulation that the latter protects

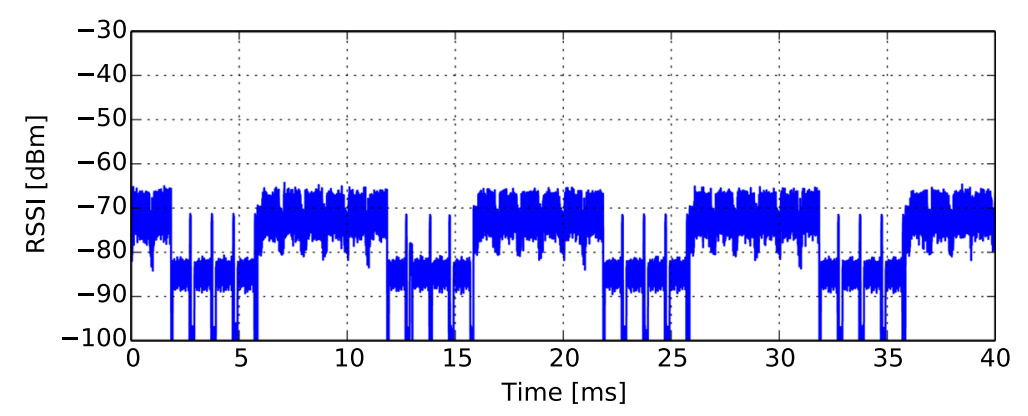

Fig. 12 TDMA WMP-WiFi over LTE-U pattern 


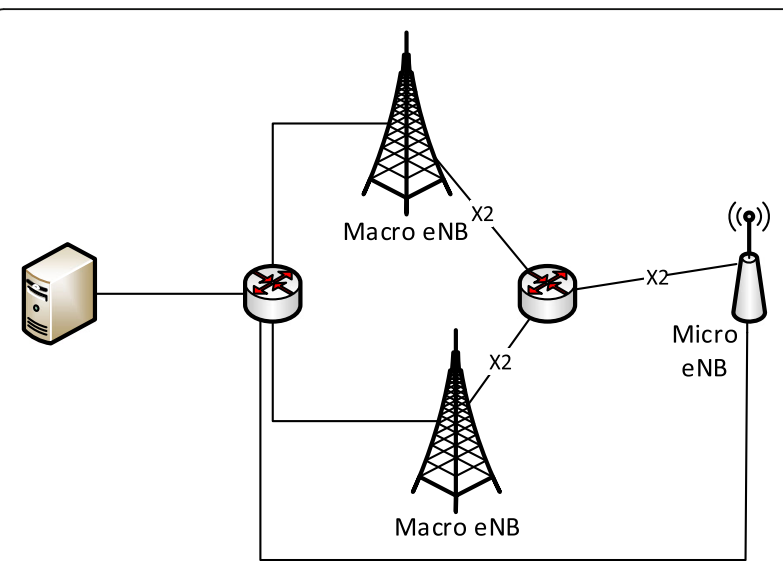

Fig. 13 Simulation scenario for Content-centric coordinated resource allocation strategies

the QoE of delay-sensitive users, without sacrificing network efficiency (i.e., the overall throughput).

The simulation scenario includes three eNBs, namely two macros and one micro. eNBs are deployed at a distance of $500 \mathrm{~m}$ and are connected via the X2 interface, as we show in Fig. 13. During the simulation, each eNB serves 30 UEs. Real-time and best-effort traffics are generated at a remote server, where applications alternate active and silence periods. During active periods, constant-size packets are generated periodically. The remote server is connected to the eNBs using dedicated connections, i.e., the evolved packet core (EPC) is not modeled. The best-effort traffic is transmitted towards the UEs attached to the macro eNBs. The real-time traffic instead is transmitted to the ones under the micro

Table 1 Main simulation parameters

\begin{tabular}{lll}
\hline Category & Parameter & Value \\
\hline $\begin{array}{l}\text { Radio } \\
\text { parameter }\end{array}$ & RBs & 15 \\
$\begin{array}{l}\text { Sim. } \\
\text { environment }\end{array}$ & \# independent replicas & 3 \\
& Simulation duration & 100 \\
& Warmup period & 10 \\
& UEs & 30 for each eNB \\
& eNBs & 3 \\
Real-time traffic: & Packet size & 50 bytes \\
& Inter-packet time & 20 ms (during active periods) \\
& Active period duration & $1 \mathrm{~s}$ \\
& Silence period duration & $5 \mathrm{~s}$ \\
& Packet size: & $\{500,1000,1500\}$ bytes \\
Best-effort & Inter-packet time & 100 ms (during active \\
traffic & Active period duration & Weibull distributed, mean 0.8 \\
& Silence period duration & Weibull distributed, mean 1.0 \\
\hline
\end{tabular}

eNB, which acts as the hotspot. A summary of the main simulation parameters is provided in Table 1 . Three load levels, namely low, medium, and high, are obtained by setting the packets size of best-effort traffic, respectively to 500,1000 , and 1500 bytes.

We simulated the above scenario using SimuLTE [39, 40], a system level simulator of LTE networks. SimuLTE is a discrete-event simulator developed for the OMNeT++ framework [41]. It simulates the data plane of the LTE/ LTE-A RAN, including all the relevant layers of the LTE protocol stack, i.e., a packet data convergence protocol radio resource control (PDCP-RRC), radio link control (RLC), MAC, and PHY. SimuLTE also includes models for macro-, micro-, and pico-eNBs, providing both isotropic and anisotropic radiation profiles, functions for MAC-level scheduling in downlink and uplink directions and X2-based communications among eNBs. The simulator leverages the INET library [42], to incorporate validated models of well-known Internet protocols (therein including SCTP for the X2, IP, TCP, and UDP) and internet nodes (e.g., the remote server).

Figure 14 shows the application delay experienced by the hotspot users, obtained with the CoCo approach (see expression (2)) using low- and high-boost levels, i.e., with $\alpha$ equal to 5 and 10 , respectively. We compare it against a content-unaware dynamic CoMP CS (as per expression (1)), for three load levels. Ninety-five percent confidence intervals are negligible, hence omitted. CoCo keeps the extra delay caused by the load increase reasonably low, ensuring a seamless responsiveness of real-time applications, regardless of how high the demand in the rest of the coordinated nodes is. The MAC-level throughput experienced in the surrounding macro cells is shown in Fig. 15, exhibiting a negligible variation at all considered loads.

The proposed CoCo approach can thus exploit the future 5G architecture to obtain content information and improve the QoE of the users, reducing the application delay up to $-65 \%$ and having a negligible performance impact on the rest of the network.

\section{Conclusions}

Current mobile networks are not effective in providing the required flexibility to adapt to varying traffic needs and to provide content and communication services where and when actually needed.

In this paper, we proposed significant architectural and functional advances for the evolution towards context and content-aware networks, with the explicit aim to influence the future standardization of $5 \mathrm{G}$ systems. In particular, we highlighted the importance of the following key technology enablers:

- Context-aware reconfigurable radio platforms triggered by proper sensors and able to easily predict 


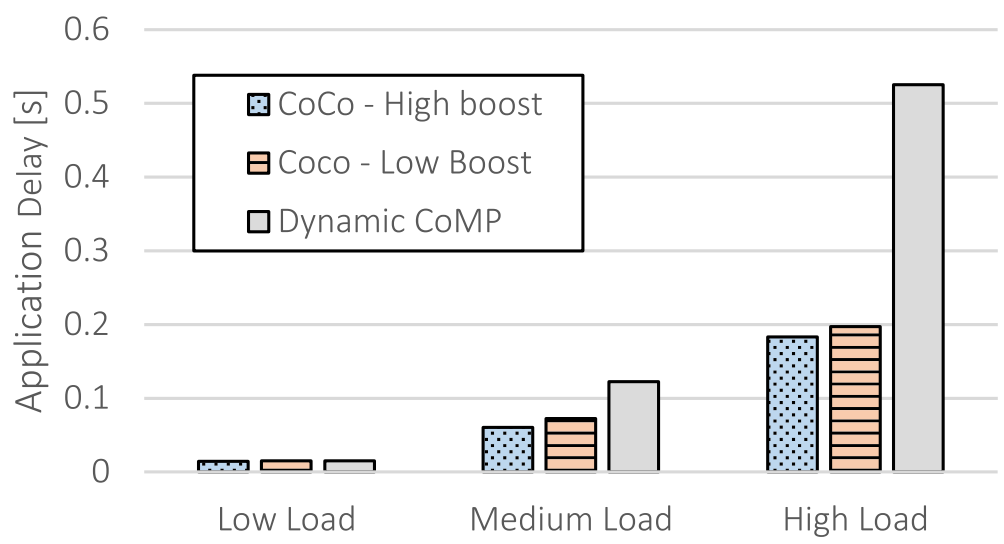

Fig. 14 Application delay with CoCO and dynamic CoMP

the interference sources in a given geographical area (e.g., by means of positioning data of the sensors) and to modify accordingly the PHY/MAC protocol to be used for D2D links (for instance, exponential backoff and rate adaptation can be disabled, or customized protocols can be downloaded to the devices to further optimize their performance);

- Content-aware CoMP (CoCo) algorithms able to exploit knowledge of the traffic content at a hotspot in order to improve the performance over traditional CoMP algorithms (unaware of the traffic content);

The above two technologies have been implemented as building blocks of the Flex5Gware network architecture. Their performance has been analyzed, through both live experiments and simulations, showing that-on the one hand-context-aware information, such as predictable interference patterns, can be detected to trigger MAC reconfiguration, thus improving coexistence of heterogeneous technologies, and-on the other-content-aware CoMP can significantly reduce the delay of real-time applications without penalizing the overall throughput.
The above two technologies can be realized in 5G networks by means of customized implementations (depending on vendors choices) or, alternatively, by exploiting content and context measures coming from standardized MEC platforms and MEC APIs. In particular, the radio network information (RNI) API currently being standardized by ETSI MEC ISG [43] has the following characteristics:

- It exposes up-to-date radio network information regarding the current radio network conditions;

- According to 3GPP specifications, this RNI (related to the user plane) is provided at the relevant granularity (e.g., per UE, cell, QCI class, or period of time);

- RNI includes information about UEs connected to the radio node(s) associated with the mobile edge host, their UE context and the related radio access bearers. Moreover, it signals changes on the above information.

All these features, when implemented in a $5 \mathrm{G}$ system, will feed suitable MEC applications dedicated to network performance and QoE improvements (as defined by

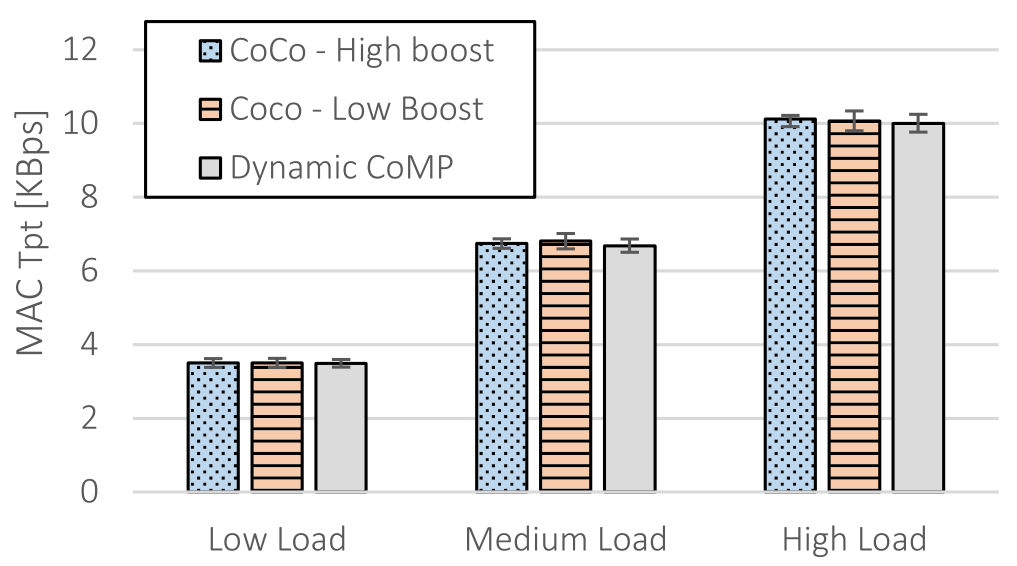

Fig. 15 MAC-level throughput with CoCo and dynamic CoMP 
ETSI MEC GS 002 [44]), where network operators and application developers will benefit from MEC RNI API (that can be seen as "middleware services" aimed at improving network performance via application-specific or generic improvements).

Researchers from the Flex5Gware project, being focused on implementation and experimental activities, are still actively contributing to ETSI MEC standard, especially for the testing and performance assessment of prototypes and advanced radio platforms [45]. In fact, this is the starting point for the evaluation of end-to-end network performance in the presence of a MEC platform and related APIs. The next step, necessary to gain a wider market acceptance of MEC technology, will be the harmonization of ETSI MEC architecture with 3GPP standard, especially in view of the definition of an interoperable interface between the MEC RNI service and 5G radio base stations.

Future work may include the implementation of a single prototype able to combine all these key technologies, as an integrated proof of concept of future flexible and reconfigurable $5 \mathrm{G}$ network platforms.

\section{Funding}

This work was supported by the European Commission in the framework of the H2020-ICT-2014-2 project Flex5Gware (Grant agreement no. 671563). The work of CTTC was partially supported by the Generalitat de Catalunya (2017 SGR 891) and by the Spanish Government under project TEC2014-58341-C4-4-R.

\section{Authors' contributions \\ The individual contributions of authors to the manuscript are specified in this section: DS was the corresponding author, integrating all the contributions and revising the paper. He contributed also to Sections 1 and 5. PS contributed to Sections 2 and 3.1. GS and AV contributed to Sections 3.3 and 4.2 and performed a general revision of the paper. IT, FG, and DG contributed to Sections 3.2 and 4.1. PV, PD, and VF contributed to the abstract sections 2 and 3 and performed a general revision of the paper; NB and MP contributed to Sections 3.1 and 3.3 and performed a general revision of the paper. All authors read and approved the final manuscript.}

\section{Competing interests}

The authors declare that they have no competing interests.

\section{Publisher's Note}

Springer Nature remains neutral with regard to jurisdictional claims in published maps and institutional affiliations.

\section{Author details}

${ }^{1}$ Intel, Neubiberg, Germany. ${ }^{2}$ Universidad Carlos III de Madrid, Madrid, Spain. ${ }^{3}$ Università di Pisa, Pisa, Italy. ${ }^{4}$ CNIT, Parma, Italy. ${ }^{5}$ WINGS ICT solutions, Kallithea, Greece. ${ }^{6} \mathrm{CTTC} / \mathrm{CERCA}$, Barcelona, Spain. ${ }^{7}$ University of Piraeus, Piraeus, Greece.

Received: 1 November 2017 Accepted: 30 July 2018

Published online: 13 August 2018

\section{References}

1. Cisco White Paper "Cisco Visual Networking Index: Global Mobile Data Traffic Forecast Update, 2016-2021", Feb. 2017, Available: http://www.cisco. com/c/en/us/solutions/collateral/service-provider/visual-networking-indexvni/mobile-white-paper-c1 1-520862.pdf. Accessed 4 Aug 2018.

2. Cisco White Paper, "Cisco Visual Networking Index: Forecast and Methodology, 2015-2020", June 2016, Available: http://www.cisco.com/c/en/ us/solutions/collateral/service-provider/ip-ngn-ip-next-generation-network/ white_paper_c11-481360.pdf. Accessed 4 Aug 2018.
3. 5G Infrastructure Association: Vision White Paper, February 2015. http://5gppp.eu/wp-content/uploads/2015/02/5G-Vision-Brochure-v1.pdf. Accessed 4 Aug 2018.

4. Flex5Gware website: http://www.flex5gware.eu/. Accessed 4 Aug 2018.

5. ETSI GS NFV-SWA 001 V1.1.1 (2014-12), Network Functions Virtualisation (NFV); Virtual Network Functions Architecture

6. ETSI white paper: "Mobile Edge Computing: A key technology towards 5G", Sept.2015; www.etsi.org/images/files/ETSIWhitePapers/etsi_wp11_mec_a_ key_technology_towards_5g.pdf. Accessed 4 Aug 2018.

7. P Marsch, Ö Bulakci, O Queseth, M Boldi, 5G System Design: Architectural and Functional Considerations and Long Term Research. Wiley, 2018

8. P Mach, Z Becvar, Mobile edge computing: a survey on architecture and computation offloading. IEEE Communications Surveys and Tutorials 19(3), 1628-1656 (2017) Q3

9. 3GPP TR 23.799, "Study on Architecture for Next Generation System", 2016

10. 3GPP TS 23.501, "System Architecture for the 5G System (Release 15)", 2017

11. I. Afolabi, et al. Network Slicing \& Softwarization: A Survey on Principles, Enabling Technologies \& Solutions. IEEE Communications Surveys \& Tutorials (2018)

12. D Thembelihle, M Rossi, D Munaretto, Softwarization of mobile network functions towards agile and energy efficient $5 \mathrm{G}$ architectures: A survey. Wirel. Commun. Mob. Comput. 2017, 21 (2017)

13. P Rost et al., Mobile network architecture evolution toward 5G. IEEE Communications Magazine 54(5), 84-91 (2016)

14. X Wang et al., Virtualized cloud radio access network for $5 \mathrm{G}$ transport. IEEE Commun. Mag. 55(9), 202-209 (2017)

15. VG Nguyen et al., SDN and virtualization-based LTE mobile network architectures: a comprehensive survey. Wireless Personal Communications Journal 86(3), 1401-1438 (2016)

16. 3GPP TS 23.402, "Architecture enhancements for non-3GPP accesses (Release 15)", 2017

17. Telecommunications Industry Association -TIA Market Report, "5G operator Survey", 2017

18. PK Agyapong et al., Design considerations for a $5 \mathrm{G}$ network architecture. IEEE Commun. Mag. 52(11), 65-75 (2014)

19. 5G-PPP, View on $5 \mathrm{G}$ Architecture (Version 2.0); https://5g-ppp.eu/wpcontent/uploads/2018/01/5G-PPP-5G-Architecture-White-Paper-Jan-2018-v2. 0.pdf. Accessed 4 Aug 2018.

20. D Sabella et al., A Flexible and Reconfigurable $5 G$ Networking Architecture Based on Context and Content Information (EuCNC, Oulu, 2017)

21. P. Marsch, et al, "5G Radio Access Network Design - A Brief Overview on the 5G-PPP Project METIS-II", EuCNC Special 5GPP session. 2017

22. P Pawelczak, K Nolan, L Doyle, SW Oh, D Cabric, Cognitive radio: ten years of experimentation and development. IEEE Communications Magazine 49(3), 90-100 (2011)

23. "Virtualization for small cells: Overview", Small Cell Forum, Release 7, http:// www.scf.io/en/documents/106_Virtualization_for_small_cells_Overview.php. Accessed 4 Aug 2018.

24. Tinnirello, I. et al., Wireless MAC processors: Programming MAC protocols on commodity Hardware. Proc. IEEE INFOCOM 2012, pp.1269-1277

25. B Jooris, J Bauwens, P Ruckebusch, P De Valck, C Van Praet, I Moerman, E De Poorter, TAISC: a cross-platform mac protocol compiler and execution engine. Computer Networks 107, 315-326 (2016)

26. Almeida, E., et al. Enabling LTE/WiFi coexistence by LTE blank subframe allocation. Proc. O IEEE ICC 2013, pp.5083-5088

27. A. M. Cavalcante et al., Performance "Evaluation of LTE and Wi-Fi Coexistence in Unlicensed Bands" 2013 IEEE 77th vehicular technology conference (VTC spring), Dresden, 2013, pp. 1-6.

28. Yubing Jian, Chao-Fang Shih, Bhuvana Krishnaswamy, Raghupathy Sivakumar. "Coexistence of Wi-Fi and LAA-LTE: experimental evaluation, analysis and insights." IEEE International Conference Communication Workshop (ICCW), 2015

29. NGMN, "Suggestions on Potential Solutions to C-RAN by NGMN Alliance," Jan 2013. https://www.ngmn.org/fileadmin/ngmn/content/downloads/ Technical/NGMN_CRAN_Suggestions_on_Potential_Solutions_to_CRAN.pdf. Accessed 4 Aug 2018.

30. G Nardini, A Virdis, G Stea, Modeling X2 Backhauling for LTE-Advanced and Assessing its Effect on CoMP Coordinated Scheduling (IWSLS2, Vienna, 2016)

31. N lardella et al., Flexible dynamic Coordinated Scheduling in Virtual-RAN deployments (FlexNets, Paris, 2017)

32. G Nardini, G Stea, A Virdis, A Frangioni, L Galli, D Sabella, GM Dell'Aera, Scalability and energy Eefficiency of Coordinated Scheduling in cellular Networks towards 5 G (CLEEN, Turin, 2017) 
33. Huawey White Paper "Cloud RAN \& The Next Generation Mobile Network Architecture", 2017.

34. 3GPP, Technical Specification Group Radio Access Network, Study on New Radio Access Technology, Radio Access Architecture and Interfaces (Release 14), 3GPP TR 38.801 V1.0.0, 2016.

35. N. Bartzoudis et al., "Energy footprint reduction in $5 \mathrm{G}$ reconfigurable hotspots via function partitioning and bandwidth adaptation", in the Proceedings of the 5th International Workshop on Cloud Technologies and Energy Efficiency in Mobile Communication Networks (CLEEN 2017), Turin, 2017.

36. SH Ali, VCM Leung, Dynamic frequency allocation in fractional frequency reused OFDMA networks. IEEE Trans. Wirel. Commun. 8(8), 4286-4295 (2009)

37. K Hoon, H Youngnam, J Jayong, Optimal subchannel allocation scheme in multicell OFDMA systems. Proc. of VTC Spring'04 3, 1821-1825 (2004)

38. L. Fang, X. Zhang, Optimal Fractional Frequency Reuse in OFDMA Based Wireless Networks, Proc. WiCOM '08, 2008, pp.1-4

39. A Virdis, G Stea, G Nardini, SimuLTE: A Modular System-level Simulator for LTE/ LTE-A Networks based on OMNeT++ (SimulTech, Vienna, 2014)

40. SimulTE webpage. http://simulte.com. Accessed 4 Aug 2018.

41. OMNeT++, https://www.omnetpp.org/. Accessed 4 Aug 2018

42. INET framework for OMNeT++: https://inet.omnetpp.org/. Accessed 4 Aug 2018.

43. ETSI GS MEC 012 V1.1.1 (2017-07): "Mobile Edge Computing (MEC); Radio Network Information API"

44. ETSI GS MEC 002 V1.1.1 (2016-03): "Mobile Edge Computing (MEC); Technical Requirements"

45. ETSI GS MEC-IEG 006 V1.1.1 (2017-01): "MEC Metrics Best Practice and Guidelines"

\section{Submit your manuscript to a SpringerOpen ${ }^{\circ}$ journal and benefit from:}

- Convenient online submission

- Rigorous peer review

- Open access: articles freely available online

- High visibility within the field

- Retaining the copyright to your article

Submit your next manuscript at $\boldsymbol{\nabla}$ springeropen.com 